JACQUES IGALENS FRÉDÉRIQUE DÉJEAN

IAE, université Toulouse I

ASSÂAD EL AKREMI

LIRHE, université Toulouse I

\title{
L'influence des systèmes économiques sur la notation sociétale
}

Cette recherche aborde la diversité des systèmes économiques et ses conséquences sur la performance sociétale de l'entreprise (PSE). À partir de la typologie des modèles de capitalisme de Amable (2005), les auteurs identifient cinq groupes de pays au sein desquels ils s'intéressent aux spécificités de la PSE. Ils postulent que les résultats de la PSE sont en partie liés à l'appartenance de l'entreprise à un système économique. Les liens avec la légitimité sont ainsi abordés. Leurs résultats démontrent I'influence de la nature du système économique sur la PSE, confirmant ainsi l'importance de ce facteur pour expliquer les différences de notations sociétales. 
$\mathrm{L}$ es enjeux de la responsabilité sociale des entreprises, s'ils ne sont pas encore clairement identifiés, sont toutefois reconnus par les acteurs économiques. L'évolution des modes de gouvernance renforce le pouvoir de nouveaux acteurs (investisseurs socialement responsables, ONG, associations). Ceux-ci mettent désormais en jeu la responsabilité des entreprises devant l'opinion publique mais aussi devant les marchés financiers. L'adoption de stratégies socialement responsables s'inscrit alors dans une démarche de recherche de légitimité (Long et Driscoll, 2008), définie par Suchman (1995) comme « la perception générale que les actions d'une entité sont désirables, convenables et adéquates par rapport à un système de normes, de valeurs et de croyances sociales » (Suchman, 1995, p. 574). La légitimité organisationnelle recouvre alors trois formes: la légitimité pragmatique, obtenue par la satisfaction des demandes des parties prenantes immédiates, la légitimité normative qui résulte d'une évaluation morale de l'organisation et la légitimité cognitive fondée sur le « taken-for-granted », ensemble d'habitudes, de pensées et de raisonnements impliquant certains modes de comportement. L'acquisition, le maintien et la restauration de légitimité reposent de plus en plus sur la capacité des entreprises à répondre aux attentes sociétales et à développer une réputation d'entreprise socialement responsable. Les engagements en termes de responsabilité sociale ou environnementale présentent souvent un caractère discrétionnaire et demandent donc à être évalués. Les agences de notation sociétale, dont la mission est d'évaluer la performance sociale des entreprises ${ }^{1}$ (PSE), ont donc un rôle à jouer dans ce processus de légitimation. Le foisonnement des travaux académiques sur la PSE rend les tentatives de définition difficiles. On peut néanmoins se référer aux travaux de Wood, en particulier l'article de 1991 dans lequel l'auteur précise que la performance sociale de l'entreprise résulte d'une « configuration organisationnelle de principes de responsabilité sociale, de processus de sensibilité sociale, de politiques et de résultats observables qui sont liés aux relations sociétales de l'entreprise » (Wood, 1991, p. 693). Cette définition souligne la nature multidimensionnelle de la notion. Elle présente surtout la performance sociétale comme étant un concept permettant d'appréhender les liens entre l'entreprise et la société civile. En effet, la PSE dépend des attentes d'un certain nombre de parties prenantes de l'entreprise et, à ce titre, ses différentes dimensions devraient être liées aux caractéristiques du système économique et social auquel l'entreprise appartient.

Le caractère émergent du marché de la notation sociétale et la divergence de certaines évaluations entraînent des interrogations quant à la pertinence des méthodologies développées par les agences de notation sociétale. Généralement, dans les systèmes de notation sociétale proposés par ces agences, si le rôle du secteur est mis en évidence, l'influence de la nature du système économique et social reste ignorée (Agle et Kelley, 2001; Ruf et al., 1998;

1. Performance Sociale de 1'Entreprise (PSE). Traduction habituelle pour le terme anglo-saxon Corporate Social Performance. Le terme « social » s'entend ici au sens de « sociétal ». 
Sharfman, 1996). Les caractéristiques du système économique et social dans lequel évolue l'entreprise ont une influence sur les attentes des parties prenantes et conditionnent de fait la PSE. Ainsi, l'intégration des caractéristiques de l'environnement économique et social de l'entreprise pourrait permettre d'expliquer les différences de notation existantes.

Dans cette étude, nous cherchons à expliquer les différences de notation sociétale. Aussi, nous analysons la diversité des systèmes économiques et sociaux et ses conséquences sur la PSE. Autrement dit, nous nous intéressons à l'influence de la diversité des systèmes économiques et sociaux sur la notation sociétale. Pour cela, nous fondons notre analyse sur l'approche développée par Amable (2005) qui distingue cinq types de capitalisme: l'économie libérale de marché, le modèle social-démocrate, le modèle européen continental, le modèle méditerranéen et le modèle asiatique. À partir de cette typologie, nous postulons qu'en fonction de leur appartenance à un système économique et social, les entreprises vont présenter des caractéristiques différentes en matière de PSE. Nous nous proposons de tester cette hypothèse en utilisant la notation extra-financière de l'agence européenne Vigeo. La notation étant un proxy de la PSE, cette dernière sera mesurée à partir des notes fournies par Vigeo.

Dans un premier temps, nous présentons les différentes variétés de systèmes économiques et sociaux. Cette typologie nous conduit à préciser les spécificités de la PSE inhérentes à chaque modèle. Le modèle de notation de Vigeo ainsi que ses implications en termes de mesure de la PSE sont développés dans une deuxième partie. La méthodologie fait l'objet de la troisième partie. La dernière est consacrée à la présentation des résultats et à leur discussion.

\section{LES DIFFÉRENTS SYSTÈMES ÉCONOMIQUES ET SOCIAUX ET LEURS IMPLICATIONS POUR LA PSE}

À l'ère de la mondialisation, la diversité des systèmes économiques et sociaux demeure. De nombreux travaux sont consacrés à l'explication de cette diversité. L'histoire, la culture, la nature et le rôle des institutions ont tour à tour été mobilisés (Albert, 1991; Aoki, 1995; Hall et Soskice, 2001; Amable, 2005).

Trois problèmes peuvent être distingués. Le premier est relatif à la mise en évidence et à la caractérisation de chacun des systèmes économiques et sociaux. Le second a trait à l'explication de ces différences et le troisième aux conséquences. Bien entendu, ces trois problèmes ne sont pas indépendants et les deux derniers sont fortement liés aux choix qui sont effectués pour caractériser les systèmes économiques et sociaux. Il est également évident que les conséquences, ou du moins certaines d'entre elles, ont des influences sur l'évolution des systèmes de sorte que chacun d'entre eux est à la fois la cause et l'effet des conséquences qu'il produit (Giddens, 1987). Notre article concerne les conséquences de la diversité des systèmes économiques et sociaux sur la notation sociétale. Si certains auteurs ont déjà relevé les conséquences de la diversité des systèmes économiques et sociaux sur le gouvernement d'entreprise (Maati, 1999), notre contribution est limitée à la performance sociale des entreprises. 
Dans la mesure où la PSE dépend des attentes de nombreux requérants ainsi que des choix et des politiques mis en place par l'entreprise pour y répondre, nous postulons que les résultats de la PSE seront en partie liés à l'appartenance de l'entreprise à un système économique et social (Pasquero, 1989) et que ces résultats renforcent les déterminants des systèmes économiques et sociaux. La figure ci-dessous est une illustration du modèle général de PSE. Nous n'étudions que les relations représentées par une flèche en traits pleins. L'impact des attentes et des pressions des requérants sur les institutions et sur les entreprises (représenté en pointillés) fait déjà l'objet de nombreuses recherches en liaison avec la théorie des parties prenantes (Freeman, 1984; Michell et al., 1997; Barnett, 2007)

Le contexte de la mondialisation a profondément marqué l'évolution des entreprises en les obligeant à innover dans tous les domaines pour faire face aux nouvelles exi- gences de compétitivité. L'innovation dépend en grande partie de l'adaptabilité des ressources humaines, c'est-à-dire à la fois de leur capacité à acquérir des compétences nouvelles et de leur mobilisation au service des objectifs de l'entreprise. Si cette double exigence est universelle, l'histoire, la culture et les institutions de chaque système économique et social forgent un cadre réglementaire normatif et cognitif particulier qui incite les entreprises à adopter des politiques sociales différentes (North, 1990). Cette première liaison est représentée par la flèche 1. Les salariés et leurs représentants, les syndicats, agissent dans le cadre d'un système de relations professionnelles à la fois contraint par les institutions sociales et permettant la reproduction voire le renforcement de ces mêmes institutions. Sous la double pression d'un cadre institutionnel et de l'action des requérants, l'entreprise effectue des choix de politique sociale, les met en œuvre et obtient des résultats; il s'agit du volet social de la PSE.

Figure 1 - Modèle général de la PSE

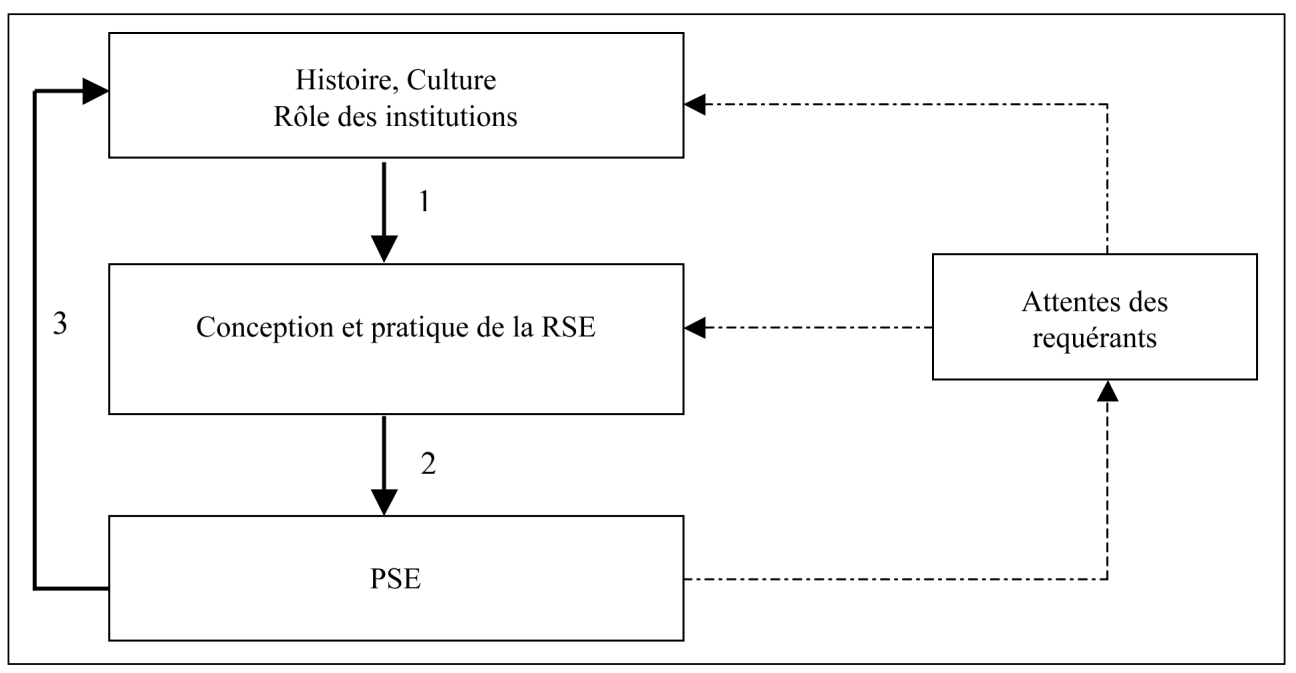


Cette seconde liaison est représentée par la flèche 2; elle illustre la recherche d'une légitimité morale au sens de Suchman (1995). Ces résultats se traduisent par un niveau d'emploi, de rémunération, par des conditions de travail qui vont, en retour, conforter les institutions et influencer les attentes des requérants salariés et syndicaux. Cette troisième liaison est représentée par la flèche 3 .

Les deux systèmes économiques et sociaux qui ont été les plus unanimement opposés par la littérature sont le système orienté marché en vigueur aux États-Unis et au Royaume-Uni et le système orienté banque en vigueur en Allemagne et au Japon (Allen et Gale, 2000). Le modèle allemand évite les tensions nées du modèle de pleine concurrence anglo-américain en associant les requérants au gouvernement d'entreprise, notamment les banques (la hausbank) et les représentants du monde du travail (Albert, 1991). Hall et Soskice évoquent la même distinction sous le terme d'économie libérale de marché et d'économie coordonnée de marché. Cette dichotomie s'appuie essentiellement sur la nature des mécanismes de coordination (Hall et Soskice, 2001). Plus précisément, ces auteurs distinguent la coordination fondée sur les forces concurrentielles du marché de la coordination centrée sur les réseaux et les relations entre les acteurs. Si la littérature sur le gouvernement d'entreprise a largement mobilisé la distinction système orienté marché versus système orienté banque, les travaux les plus récents s'orientent vers l'étude des systèmes juridiques pour expliquer les différences nationales en matière de gouvernance d'entreprise. Certains auteurs s'intéressent ainsi aux différentes formes juridiques, en particulier aux modes de pro- tection des intérêts des actionnaires minoritaires. Reprenant l'opposition classique entre le système du common-law et le système du droit civil, La Porta et al. (1998, 2000) proposent quatre clusters: les pays relevant du common-law (États-Unis et Royaume-Uni), le droit civil français, le droit civil germanique et le droit civil scandinave. Cette typologie permet d'étudier les formes de protection des intérêts des actionnaires minoritaires (La Porta et al., 1998) et leur impact, par exemple, sur la valorisation des entreprises (La Porta et al., 2002). Dans la lignée des travaux de La Porta et al. (1998), Leuz et al. (2003) comparent les pratiques de earnings management dans 31 pays. Ces gestions du résultat ont pour visée de masquer la véritable performance des entreprises et ainsi de confisquer aux actionnaires minoritaires une partie des bénéfices. À partir de la distinction common-law et droit civil, et sur la base de caractéristiques juridiques et institutionnelles identiques, les 31 pays sont regroupés en trois clusters, proches de la classification proposée par La Porta et al. (1998). Pour étudier les différences de pratiques dans la reddition des comptes, la référence aux systèmes comptables permet également de proposer des clusters pour effectuer des comparaisons internationales. Par exemple, Brown et Higgins (2001) classent les pays en fonction de trois systèmes comptables: le système américain (US GAAP), le système américain-britannique (Autralie, Hong-Kong, Pays-Bas, Afrique du Sud, Royaume-Uni) et le système européen continental (France, Allemagne, Italie, Japon, Espagne, Suède, Suisse). Dans une certaine mesure, cette typologie comptable permet d'intégrer l'hétérogénéité des systèmes économiques. 
En centrant leur analyse sur l'innovation, et en s'intéressant aux caractéristiques des formes institutionnelles des secteurs de la finance, de l'emploi et de l'éducation, d'autres auteurs ont distingué quatre types de systèmes sociaux: un modèle fondé sur le marché, un modèle méso-corporatif représentatif du Japon, un modèle socialdémocrate représentant les économies scandinaves et un modèle d'intégration européenne (Amable et al., 1997). Dans ses travaux ultérieurs, Amable (2005) distingue cinq types de capitalisme: le marché, le modèle social-démocrate, l'européen continental, le méditerranéen et l'asiatique. Dans le capitalisme de marché, la concurrence constitue l'élément essentiel. Elle rend les entreprises très réactives, notamment concernant l'emploi. Le modèle social-démocrate réagit à la pression de la concurrence par un mélange de protection modérée de l'emploi, un haut niveau de protection sociale et l'accès facile à la formation. Un système de négociations sociales coordonnées permet une fidélisation des salariés fondée sur la solidarité. Le modèle européen continental est caractérisé par un degré plus élevé de protection de l'emploi et une protection sociale moins élevée. Un système financier centralisé facilite l'élaboration de stratégies à long terme pour les entreprises. Les négociations salariales sont coordonnées et une politique des salaires fondée sur la solidarité est développée mais à un degré moindre que dans le modèle précédent. Le modèle méditerranéen est caractérisé par une protection de l'emploi plus forte et une protection sociale moins forte que dans le modèle européen continental. Enfin, le modèle asiatique dépend fortement des stratégies industrielles des grandes firmes, établies en collaboration avec l'État, et d'un système financier centralisé qui permet le développement de stratégies à long terme.

Selon Amable, les cinq modèles de capitalisme se distinguent à partir de la combinaison des cinq dimensions suivantes : les marchés de produits et de services, le marché du travail, le système financier, la protection sociale et l'éducation (Amable, 2005, p. 223-224). Pour cette recherche, nous avons retenu cette dernière approche. À cet égard, la combinaison de ces cinq dimensions telle qu'elle est formulée permet, dans une certaine mesure, d'appréhender les différents volets de la PSE. En outre, les typologies antérieures, pourtant largement utilisées pour étudier les formes de gouvernance d'entreprise et de nombreuses pratiques comptables, nous ont semblé incomplètes. En effet, si ces travaux reconnaissent la nécessité d'intégrer l'hétérogénéité des systèmes économiques, ils attachent toutefois davantage d'importance aux caractéristiques des systèmes juridiques et aux règles comptables, conduisant ainsi à laisser de côté certaines dimensions de la PSE. Par ailleurs, les cinq modèles de capitalisme décrits par Amable (2005) restent proches de la typologie proposée par La Porta et al. (1998) et reprise dans de nombreux travaux en finance de marché.

L'auteur de cette typologie a testé sa pertinence sur les 21 pays de l'OCDE dans les années 1990. Bien qu'il remarque qu'un pays donné ne relève pas systématiquement du même modèle pour chaque dimension étudiée, son analyse aboutit à la correspondance suivante entre modèle et pays (tableau 1). 
En partant des caractéristiques principales de chacun des modèles de capitalisme proposés par Amable, nous avons postulé des différences en matière de PSE, différences fondées sur les particularités de chaque modèle. Ainsi, nous cherchons à démontrer que les choix et les résultats des entreprises demeurent fortement déterminés par le modèle de capitalisme auquel elles appartiennent.

Tableau 1 - Typologie des systèmes économiques et sociaux

\begin{tabular}{|c|c|c|}
\hline Type de capitalisme & Caractéristiques principales & Pays \\
\hline Libéral de marché & $\begin{array}{l}\text { - Marchés de produits déréglementés } \\
\text { - Flexibilité du marché du travail } \\
\text { - Système financier et gouvernement } \\
\text { d'entreprise fondés sur le marché } \\
\text { - Modèle libéral de protection sociale } \\
\text { - Système éducatif concurrentiel }\end{array}$ & $\begin{array}{l}\text { - Royaume-Uni } \\
\text { - États-Unis } \\
\text { - Canada } \\
\text { - Australie }\end{array}$ \\
\hline Européen continental & $\begin{array}{l}\text { - Marchés de produits faiblement réglementés } \\
\text { - Marchés du travail coordonnés } \\
\text { - Système financier fondé sur les institutions } \\
\text { financières } \\
\text { - Modèle corporatiste de protection sociale } \\
\text { dans lequel les allocations sont fondées sur } \\
\text { l'emploi } \\
\text { - Système éducatif public }\end{array}$ & $\begin{array}{l}\text { - France } \\
\text { - Allemagne } \\
\text { - Autriche } \\
\text { - Belgique } \\
\text { - Irlande } \\
\text { - Norvège } \\
\text { - Suisse } \\
\text { - Pays-Bas }\end{array}$ \\
\hline Social-démocrate & $\begin{array}{l}\text { - Marchés de produits réglementés } \\
\text { - Marchés du travail réglementés } \\
\text { - Système financier constitué par des banques } \\
\text { - Modèle universel de protection sociale } \\
\text { - Système éducatif public }\end{array}$ & $\begin{array}{l}\text { - Suède } \\
\text { - Finlande } \\
\text { - Danemark }\end{array}$ \\
\hline Asiatique & $\begin{array}{l}\text { - Concurrence « dirigée » plutôt que } \\
\text { réglementée } \\
\text { - Marchés du travail réglementés } \\
\text { - Système financier constitué par des banques } \\
\text { - Faible niveau de protection sociale } \\
\text { - Système éducatif privé }\end{array}$ & $\begin{array}{l}\text { - Corée } \\
\text { - Japon }\end{array}$ \\
\hline Méditerranéen & $\begin{array}{l}\text { - Marchés de produits réglementés } \\
\text { - Marchés du travail réglementés } \\
\text { - Système constitué par des banques } \\
\text { - Protection sociale limitée } \\
\text { - Système éducatif faible }\end{array}$ & $\begin{array}{l}\text { - Italie } \\
\text { - Espagne } \\
\text { - Portugal } \\
\text { - Grèce }\end{array}$ \\
\hline
\end{tabular}

Source: Amable (2005). 


\section{PRÉSENTATION DU MODÈLE VIGEO}

La notion de PSE recouvre de nombreux sens, de la gestion des dimensions de la RSE (Wood, 1991) à la réponse aux pressions sociétales dans une perspective d'adaptation à l'environnement (Zeniseck, 1979). Elle est souvent assimilée à un instrument de légitimation du système capitaliste (Pasquero, 2005; Jones, 1996). Le recours au système de notation extra-financière, ou sociétale, développé par des agences spécialisées est désormais fréquent pour mesurer la PSE. Cela est notamment le cas pour les travaux explorant le lien entre la PSE et la performance financière (Margolis et Walsh, 2003; Rowley et Berman, 2000; Griffin et Mahon, 1997). L'utilisation de ces notations suscite des interrogations quant à la pertinence de la construction des bases de données (Gond, 2006; Déjean et Gond, 2004). En particulier, Wood et Jones (1995) remarquent que les modèles des agences de notation extrafinancière ne sont que faiblement cohérents avec les modèles théoriques de la PSE et ne sont pas exempts de toute dimension idéologique. Par exemple, l'agence de notation américaine KLD propose un modèle fondé sur une approche de type screening négatif: des critères sur le caractère controversé de certaines activités permettent de mesurer le degré d'implication des entreprises dans ces secteurs (alcool, armement, jeux de hasard, industrie militaire et nucléaire, tabac). Cette approche binaire ne permet pas d'appréhender le caractère multidimensionnel de la PSE (Igalens et Gond, 2005). De ce fait, afin d'utiliser une mesure cohérente avec les spécificités de la PSE explicitées dans la première partie, nous avons souhaité exclure tout modèle fondé sur une approche binaire et retenu le modèle de l'agence européenne Vigeo. L'utilisation de ce modèle implique que cette recherche n'échappe pas aux critiques formulées à l'encontre des travaux mobilisant les bases de données sur la PSE. Toutefois, l'analyse du processus de construction de la notation sociétale ne constitue pas l'objet de la présente recherche.

Vigeo mesure la PSE à partir d'objectifs de responsabilité sociale déclinés en six domaines. Ces domaines sont issus des cinq domaines qui existaient à l'époque d'ARESE : environnement, RH, clientsfournisseurs, actionnaires et société civile qui ont été en partie rebaptisés, par exemple « actionnaires » est devenu « gouvernement d'entreprise», " clients-fournisseurs » est devenu " comportement sur les marchés » et «société civile» est devenu « engagement sociétal » tandis que la nouvelle présidente, N. Notat, ajoutait « droits humains ». À l'origine, les cinq domaines d'ARESE étaient inspirés des autres agences et notamment KLD et SAM. Au total, le référentiel d'évaluation des entreprises est composé de 37 critères génériques répartis en six domaines :

1) Ressources humaines: amélioration continue des relations professionnelles, des relations d'emploi et des conditions de travail.

2) Droits humains : respect de la liberté syndicale et de la promotion de la négociation collective, non-discrimination et promotion de l'égalité, élimination des formes de travail proscrites, prévention des traitements inhumains ou dégradants de type harcèlement sexuel, protection de la vie privée et des données personnelles. 
3) Environnement: protection, sauvegarde, prévention des atteintes à l'environnement, mise en place d'une stratégie managériale appropriée, écoconception, protection de la biodiversité.

4) Comportement sur les marchés: prise en compte des droits et intérêts des clients, intégration de standards sociaux et environnementaux dans la sélection des fournisseurs et sur l'ensemble de la chaîne d'approvisionnement, prévention effective de la corruption, respect des règles concurrentielles.

5) Gouvernement d'entreprise: efficience et probité, effectivité et efficience des mécanismes d'audit et de contrôle, transparence et rationalité de la rémunération des dirigeants.

6) Engagement sociétal: intégration managériale de l'engagement, contribution au développement économique et social des territoires d'implantation et de leurs communautés humaines, contribution transparente et participative à des causes d'intérêt général.

Le modèle d'analyse de Vigeo recourt aux principes de gestion de la qualité totale autour d'un référentiel baptisé « $\mathrm{L}-\mathrm{D}-\mathrm{R} »$, pour « Leadership ou Politique - Déploiement - Résultats », qui est une adaptation libre des trois premières étapes de la fameuse « roue de Deming » (Plan, Do, Check, Act). Ce référentiel sera mobilisé de manière transversale pour configurer les modalités de construction des notes sur les six domaines. Ainsi, Vigeo analyse la pertinence des politiques (visibilité, contenu, portage), la cohérence du déploiement (processus, moyens, reporting) et l'efficacité des résultats (indicateurs, points de vue des parties prenantes, controverse).
Concernant les méthodes de travail, la méthodologie repose en grande partie sur des analyses statistiques et l'objectif prioritaire est de minimiser le travail d'évaluation subjective de l'analyste et d'objectiver au maximum le processus. L'inégale disponibilité de données quantifiées dans un format standardisé pour chaque critère va conduire les analystes à mettre au point une méthode de scoring s'inspirant des modèles de qualité et de l'analyse financière. Les informations disponibles sont quantifiées en cochant des cases et en estimant des ratios. La méthode articule pour chaque critère d'évaluation deux grandes phases: 1) une phase de scoring visant à transformer les informations qualitatives en informations quantitatives et 2) une phase de ranking, consistant à classer les entreprises les unes par rapport aux autres en fonction des différents scores au sein d'un même secteur.

Le processus de scoring est construit de manière à se rapprocher au maximum d'un processus automatique où l'analyste « laisse s'exprimer » les chiffres qu'il recueille ou renseigne directement le référentiel en cochant des cases ou en inscrivant des chiffres, étant donné l'information dont il dispose. Ce processus est baptisé par les analystes « traitement automatique de l'information ». De manière idéale, il doit conduire à faire en sorte que le référentiel puisse s'actualiser automatiquement à partir des informations chiffrées recueillies à partir de différentes sources, sans intervention de l'analyste.

Le second «bloc » de la méthodologie ranking est un processus d'analyse statistique qui s'appuie sur ces scores pour établir un classement relatif des entreprises les unes par rapport aux autres au sein d'un 
même secteur et pour un critère donné. Ce classement est ensuite subdivisé en quartiles et les notes (sous la forme,,$+++=,-$, - -) sont finalement attribuées en fonction de l'appartenance de l'entreprise à l'un des quatre quartiles (A pour le premier, B pour le second, etc.).

À partir des six dimensions du modèle Vigeo et sur la base de la typologie développée par Amable (2005), nous formulons cinq hypothèses visant à caractériser la PSE en fonction des variétés des systèmes économiques et sociaux.

1) Concernant le gouvernement d'entreprise, la défense des actionnaires vis-à-vis des dirigeants est mieux assurée par les marchés financiers des économies libérales (La Porta et al., 1998; 2000; 2002; Leuz et al. 2003). Tout un dispositif d'incitations et de contrôle est mis en place: indicateurs de création de valeur, stock-options, offres publiques d'achat ou d'échanges, indépendance des autorités de marché. Ainsi, les conditions d'une forte protection des actionnaires notamment minoritaires sont assurées. Les économies du modèle européen continental et du modèle méditerranéen n'ont pas les mêmes règles, le marché pour le contrôle des entreprises est beaucoup moins actif, les banques ont parfois deux fonctions, prêteurs de fonds et actionnaires de référence, ce qui est susceptible de brouiller la clarté des rôles, notamment les relations d'agence entre propriétaires et dirigeants. Le modèle social démocrate apparaît entre les deux, moins performant que le modèle libéral mais plus performant que les économies du modèle européen continental et du modèle méditerranéen (La Porta et al., 1997; 1998).

Hypothèse 1. Sur le volet gouvernement d'entreprise, les entreprises relevant du capitalisme libéral de marché obtiendront une meilleure performance que les autres entreprises.

2) L'engagement sociétal comprend les actions menées par l'entreprise dans l'intérêt des populations et des territoires sur lesquels elle est engagée, qu'il s'agisse des populations des pays en développement ou de territoires menacés notamment d'un point de vue environnemental. Mais l'engagement sociétal comprend également différentes formes de philanthropie, le don à de grandes causes d'intérêt général (recherche, santé, culture) ainsi que des formes plus récentes telles que le volontariat des salariés vis-à-vis de ces mêmes grandes causes (bénévolat de compétences).

Le capitalisme fondé sur le marché semble moins porté que les autres à l'engagement sociétal du fait des exigences de rentabilité du marché financier et, sauf exception, de la faible rentabilité des actions qui constituent l'engagement sociétal de l'entreprise (Cummings, 2000; Teoh et al., 1999). Mais la tradition philanthropique du capitalisme protestant va totalement à l'encontre d'une telle affirmation. Comme le montre Heald (1970), le concept de responsabilité sociale apparaît dans les discours des dirigeants à partir de la fin du XIX $^{\mathrm{e}}$ et au début du $\mathrm{XX}^{\mathrm{e}}$ siècle aux États-Unis. Cette responsabilité s'incarna tout d'abord dans le développement d'activités philanthropiques. Dès 1889, Carnegie, immigrant écossais qui devint à travers sa fortune l'un des symboles du rêve américain, publia un document qui deviendra une référence en matière de philanthropie. Dans son « Évangile des riches » (The Gospel of Wealth), il développa une série de réflexions relatives aux responsabilités des riches hommes d'affaires de l'époque, considérant qu'il 
était de leur devoir de mener une vie non ostentatoire et que les surplus de richesse dont ils bénéficiaient devaient être gérés et redistribués en vue de l'intérêt public (voir Heald, 1970, p. 17-18).

Durant la première moitié du $\mathrm{XX}^{\mathrm{e}}$ siècle, les premiers discours et théorisations de la responsabilité sociale seront largement marqués par les concepts protestants de public service et de stewardship. Ces concepts reprennent l'idée d'un contrat implicite caractérisant la relation entre l'entreprise et la société. On retrouve la conviction que la propriété n'a rien d'un droit absolu et inconditionnel et qu'elle ne peut être justifiée que dans la mesure où l'administration privée des biens permet d'accroître le bien-être de la communauté (Acquier et al., 2005).

Pour cette raison, nous postulons que le capitalisme fondé sur le marché sera le plus performant concernant la dimension sociétale. Concernant les autres types de capitalisme, le modèle européen continental et le modèle méditerranéen devraient être mieux placés que le capitalisme social-démocrate $\mathrm{du}$ fait de l'existence d'ex-empires coloniaux (Allemagne, Belgique, France pour le modèle continental et Espagne, Italie, Portugal pour le modèle méditerranéen). Le modèle social-démocrate (Danemark, Finlande, Suède) n'ayant pas une histoire comparable, les engagements vis-à-vis de territoires ou de populations de certains pays en développement ne seront pas marqués des mêmes contingences. Notre hypothèse $\mathrm{H} 2$ consiste alors à définir trois niveaux d'engagement envers la société civile.

Hypothèse 2. Concernant l'engagement des entreprises envers la société civile, le niveau d'engagement sera élevé pour l'éco- nomie de marché (du fait de la tradition philanthropique), intermédiaire pour les modèles méditerranéen et continental (du fait de l'histoire coloniale) et faible pour l'économie sociale-démocrate.

3) Deux types de capitalisme s'opposent fortement concernant la dimension comportement sur les marchés: le capitalisme de marché et le capitalisme social-démocrate. Dans le capitalisme libéral de marché, une grande importance est accordée à la concurrence par les prix: l'État reste neutre sur les conditions des échanges des produits et services. La protection des clients repose sur leur capacité d'auto-organisation: les mouvements de consommateurs par exemple. Aucune protection ou défense des intérêts des fournisseurs n'est engagée. Dans les économies socialesdémocrates, il y a au contraire un fort engagement de l'État sur les marchés de produits et un haut degré de coordination d'autres signaux que les prix. L'intérêt des clients peut être totalement pris en charge par des structures publiques ou indirectement favorisé par des appuis à des mouvements dirigés vers la défense de la qualité ou vers des catégories sociales telles que les « petits producteurs » ou les «producteurs locaux ». Autrement dit, plus l'économie a dérégulé ses marchés de biens et services et moins les entreprises prennent en compte les intérêts de long terme des clients et des fournisseurs.

Dans le capitalisme européen continental et dans le capitalisme méditerranéen, les entreprises se situent entre ces deux extrêmes: importance modérée de la concurrence par les prix et importance relativement élevée de la concurrence par la qualité. Les autorités publiques s'engagent 
moins que dans les économies socialeslibérales et il y a notamment moins de protection contre les sous-traitants ou les fournisseurs étrangers.

Hypothèse 3. Sur le volet comportement sur les marchés, les entreprises relevant du capitalisme social-démocrate seront plus performantes que les entreprises relevant du capitalisme libéral de marché.

4) Concernant le volet social de la PSE, qu'il s'agisse de la politique sociale en matière de $\mathrm{RH}$ ou du respect des droits humains, l'hypothèse $\mathrm{H} 4$ tient compte à la fois des particularités des marchés du travail et de la protection sociale. Dans le capitalisme de marché, la protection de l'emploi est très faible, la flexibilité est forte, le recours au travail temporaire aisé. Aucune politique de l'emploi ne vient contre-balancer les à-coups du marché financier, la négociation salariale est souvent décentralisée et les syndicats sont sur des positions plus défensives qu'offensives. Pour ces raisons, sur le volet social, la PSE reste faible. Dans le modèle méditerranéen, il existe souvent une protection de l'emploi mais qui reste limitée aux grandes entreprises. Le marché du travail est dual et la plus grande partie des travailleurs est, en réalité, très exposée aux fluctuations du marché. Ces travailleurs sont en effet employés par des entreprises de petite et moyenne taille non protégées par les pouvoirs publics. Les conflits du travail sont plus nombreux que dans les autres capitalismes, il n'y a pas de politique du travail active et le niveau de protection sociale est modéré car la structure des dépenses sociales de l'État penche vers le soulagement de la pauvreté plus que vers la protection sociale des actifs. Pour des raisons différentes de celles qui préva- lent dans le cas du capitalisme de marché, nous émettons l'hypothèse d'un score faible sur le volet social de la PSE pour les entreprises du modèle méditerranéen.

Les marchés du travail réglementés vont souvent de pair avec une importance accordée à la place des syndicats dans les relations de travail, ce qui se traduit par exemple par des négociations centralisées ou coordonnées. Il s'agit là du cas de figure le plus favorable aux syndicats et donc par conséquent aux salariés qui bénéficient d'accords bien négociés. Que cette place soit liée à des dispositions institutionnelles (en Allemagne et en France par exemple) ou à un fort taux d'adhésion syndicale (au Danemark, en Finlande, en Norvège ou en Suède), les variétés du capitalisme à marché du travail réglementé ou coordonné devraient être, sur cet axe, plus performantes que le capitalisme libéral de marché ou le capitalisme méditerranéen, le capitalisme social-démocrate se distinguant par un modèle très protecteur et universel tandis que le capitalisme européen continental réserve les allocations principalement à ceux qui sont engagés dans l'emploi. Notre hypothèse concernant le volet social de la PSE consiste donc à placer les variantes européenne continentale et sociale-démocrate avant les variantes libérales de marché et méditerranéenne.

Hypothèse 4. Les entreprises relevant du capitalisme européen continental et socialdémocrate sont plus performantes concernant le volet social de la PSE que celles relevant du capitalisme libéral de marché et du capitalisme méditerranéen.

5) La dimension environnementale ne doit pas être considérée avec la logique de la dimension sociale car le marché financier 
reconnaît depuis quelques années les risques environnementaux alors qu'il ne reconnaît pas les risques sociaux. Le marché financier contraint donc les entreprises relevant du capitalisme libéral de marché à tenir compte de ces risques par des mécanismes de prévention et d'assurance. Traditionnellement, les pays nordiques correspondant au modèle social-démocrate ont une sensibilité environnementale marquée. En revanche, le modèle continental semble moins sensibilisé (à l'exception il est vrai de l'Allemagne) et le modèle méditerranéen demeure en retard dans la prise en compte des facteurs écologiques. Il est vrai que le problème particulier du réchauffement climatique a tendance à faire converger les variétés du capitalisme notamment par la mise en place du marché des « droits à polluer » calculés à partir des émissions de gaz à effet de serre. Mais le réchauffement climatique et ses conséquences ne constituent pas à eux seuls la totalité de la dimension environnementale, les problèmes de pollution, l'épuisement des ressources naturelles ont également une importance croissante concernant les décisions des entreprises. Pour ces raisons, nous émettons l'hypothèse de deux modèles dominants concernant la dimension environnementale (les modèles libéral de marché et social-démocrate), d'un modèle intermédiaire (le modèle continental) et d'un modèle à la traîne (le modèle méditerranéen).

Hypothèse 5. Sur le volet environnement, les entreprises relevant du système capitaliste de marché seront plus performantes que les entreprises relevant du capitalisme méditerranéen.

Un récapitulatif sur les liens entre les modèles de capitalisme et les caractéristiques de la PSE est repris dans le tableau 2.

Tableau 2 - Liens entre les systèmes de capitalisme et les types de PSE construits

\begin{tabular}{|c|c|}
\hline Type de capitalisme & Caractéristiques de la PSE \\
\hline Libéral de marché & $\begin{array}{c}\text { Une forte importance est accordée aux dimensions } \\
\text { gouvernance, engagement sociétal et environnement }\end{array}$ \\
\hline Européen continental & Une forte importance est accordée au volet social \\
\hline Social démocrate & $\begin{array}{r}\text { Une forte importance est accordée aux dimensions } \\
\text { comportement sur les marchés et sociale }\end{array}$ \\
\hline Méditerranéen & $\begin{array}{c}\text { Une importance modérée est accordée aux dimensions } \\
\text { engagement sociétal et comportement sur les marchés }\end{array}$ \\
\hline
\end{tabular}




\section{MÉTHODOLOGIE}

\section{Les données}

L'étude porte sur les entreprises européennes listées au DJ STOXX 600 ou au SBF 120 sur une période de deux ans, entre janvier 2004 et décembre 2005. Dans le modèle de Vigeo, les six dimensions décrites précédemment font l'objet d'une analyse dite par angle d'approche ou L - D - R (Leadership, Déploiement et Résultats). Il s'agit d'évaluer distinctement la politique annoncée par l'entreprise (le leadership), puis ses efforts en termes de processus et de moyens mis en œuvre pour appliquer cette politique (le déploiement) et enfin les résultats qu'elle obtient en la matière (le résultat). La note finale est le produit équi-pondéré des scores attribués à ces trois angles d'approche. Au total, pour chaque score (score L, score D, score R et score final), nous disposons de 4284 observations sur les deux années.

Les entreprises analysées appartiennent à 17 pays et 35 secteurs d'activité. Ces pays ont été regroupés afin de mettre en évidence les différences de variétés des capitalismes. Au vu du nombre d'observations disponibles pour chacun des groupes, nous avons constitué cinq échantillons de taille comparable:

- premier groupe: Danemark, Finlande, Suède, Norvège,

- deuxième groupe: Belgique, Luxembourg, Pays-Bas, Autriche, Allemagne, Suisse,

- troisième groupe: Portugal, Espagne, Italie, Grèce,

- quatrième groupe: France,

- cinquième groupe: Angleterre, Irlande.

Selon la typologie proposée par Amable (2005), le premier groupe correspond au modèle social-démocrate (à l'exception de la Norvège, classée dans le modèle européen continental). Les deuxième et quatrième groupes caractérisent le modèle européen continental. Le modèle méditerranéen est représenté par le troisième groupe. Au sein du modèle européen continental, nous avons souhaité isoler la France (quatrième groupe), le nombre d'observations disponibles permettant d'obtenir un échantillon de taille comparable. Le cinquième groupe est quant à lui représentatif de l'économie libérale de marché. Étant donné le faible nombre d'observations dont nous disposions, nous n'avons pas retenu les entreprises américaines, de même que les entreprises japonaises (le modèle asiatique ne sera donc pas testé).

\section{Les analyses}

Nous avons procédé à une série d'analyses pour étudier l'effet de l'appartenance à un modèle de capitalisme sur les scores de performance sociale, à savoir les scores L - D - R et le score global. Nous avons commencé par une série d'analyses univariées de la variance (Anova), en utilisant comme variable dépendante le score de performance sociale et comme facteur, l'appartenance du pays à un modèle de capitalisme. Une série de tests d'homogénéité des variances (test de Levene) et d'égalité des moyennes (test de Welch) ont permis à chaque fois de s'assurer de la pertinence de l'analyse de la variance. Deuxièmement, nous avons utilisé un modèle linéaire général (GLM) pour étudier simultanément l'effet du secteur et l'effet du pays sur le score de performance 
sociale des entreprises afin de voir si en contrôlant pour le facteur « secteur d'activité », l'effet de l'appartenance du pays à un modèle capitaliste reste significatif. Ces analyses nous ont ainsi permis de nous assurer que les résultats obtenus ne sont pas influencés par le poids des différents secteurs d'activité. Nous avons aussi effectué une série de tests post-hoc de Scheffé afin de s'assurer que cet effet reste aussi significatif si on compare a posteriori les groupes de pays deux à deux.

Les données de Vigeo ont ainsi été analysées sur la base d'une série d'Anova et d'un modèle linéaire général pour contrôler l'effet du secteur d'activité. Nous nous sommes intéressés aux scores de chaque niveau (L - D - R) et au score global des cinq groupes de pays. Les résultats montrent que les scores moyens sont significativement différents en fonction de l'appartenance du pays à des modèles distincts de capitalisme. De plus, l'étude des moyennes marginales estimées indique que lorsque le secteur est contrôlé, les scores moyens obtenus sont sensiblement proches de ceux que l'on obtient en l'absence du contrôle de cette variable. Aussi, l'effet du pays reste souvent significatif dans les tests post-hoc.

\section{RÉSULTATS : PRÉSENTATION ET DISCUSSION}

Les résultats des Anova sont présentés successivement dans les tableaux 3 à 8 en fonction du critère de notation: gouvernement d'entreprise, engagement social, comportement sur les marchés, ressources humaines et droits de l'homme, enfin environnement. Chaque tableau présente les différentes statistiques de significativité ainsi que les scores moyens des différents groupes de pays; ces scores sont notés de 0 à 100 et classés par ordre croissant.

Les résultats présentés dans les tableaux 3 à 8 apportent quelques enseignements sur les systèmes économiques et sociaux. Ils témoignent d'une forte disparité entre le capitalisme libéral de marché (groupe 5), classé systématiquement au premier rang, et le capitalisme méditerranéen (groupe 3) que l'on retrouve très souvent quant à lui au dernier rang. Cela confirme le faible enga- gement, de manière générale, des pays d'Europe du Sud envers la RSE. Au contraire, les pays anglo-saxons apparaissent comme les tenants d'une approche orientée PSE. On constate aussi que le classement du groupe 1 (capitalisme socialdémocrate) est relativement constant quel que soit l'angle d'approche retenu. En revanche, au sein du modèle européen continental, des disparités apparaissent. En particulier, la France (groupe 4), moins bien classée que tous les autres pays appartenant à ce même modèle économique et social (groupe 2), possède en revanche un meilleur score R. Autrement dit, les données quantitatives fournies par les entreprises appartenant au modèle européen continental, à l'exception des entreprises françaises, ne sont pas à la hauteur ni des engagements annoncés ni des moyens d'action mis en place. Inversement, la communication des entreprises françaises apparaît en retrait par rapport aux résultats obtenus. 
Tableau 3 - Résultats de l'Anova mettant en évidence l'effet du pays sur les scores de gouvernement d'entreprise

\begin{tabular}{|c|c|c|c|c|}
\hline Effet du pays & Score global & Score L & Score D & Score R \\
\hline $\begin{array}{l}\text { Somme des carrés }= \\
23090,27\end{array}$ & Groupe $5(59,46)$ & Groupe $5(67,85)$ & Groupe $5(62,81)$ & Groupe $5(47,68)$ \\
\hline Degré de liberté = 4 & Groupe $2(40,94)$ & Groupe $2(45,42)$ & Groupe $2(43,83)$ & Groupe $4(34,39)$ \\
\hline $\begin{array}{l}\text { Carrés moyens }= \\
5772,57\end{array}$ & Groupe $1(36,26)$ & Groupe $1(37,22)$ & Groupe $1(38,78)$ & Groupe $2(33,58)$ \\
\hline Test en $\mathrm{F}=38,49$ & Groupe $4(35,09)$ & Groupe $3(37,17)$ & Groupe $3(36,40)$ & Groupe $1(32,70)$ \\
\hline Significativité $=0,00$ & Groupe $3(33,02)$ & Groupe $4(34,72)$ & Groupe $4(36,31)$ & Groupe $3(27,48)$ \\
\hline $\begin{array}{l}\text { Variance expliquée }= \\
0,51\end{array}$ & Moyenne $=44,80$ & Moyenne $=49,03$ & Moyenne $=47,53$ & Moyenne $=37,85$ \\
\hline
\end{tabular}

Tableau 4 - Résultats de l'Anova mettant en évidence l'effet du pays sur les scores de l'engagement envers la communauté

\begin{tabular}{|c|c|c|c|c|}
\hline Effet du pays & Score global & Score L & Score D & Score $\mathbf{R}$ \\
\hline $\begin{array}{l}\text { Somme des carrés }= \\
4012,62\end{array}$ & Groupe $5(43,24)$ & Groupe $5(44,34)$ & Groupe $5(41,63)$ & Groupe $5(43,96)$ \\
\hline Degré de liberté $=4$ & Groupe $4(35,96)$ & Groupe $3(38,57)$ & Groupe $4(33,21)$ & Groupe $1(38,30)$ \\
\hline $\begin{array}{l}\text { Carrés moyens }= \\
1003,15\end{array}$ & Groupe $3(35,74)$ & Groupe $4(37,30)$ & Groupe $3(33,17)$ & Groupe $4(37,43)$ \\
\hline Test en $F=3,79$ & Groupe $1(35,04)$ & Groupe $1(36,52)$ & Groupe $2(33,10)$ & Groupe $2(37,04)$ \\
\hline Significativité $=0,00$ & Groupe $2(33,67)$ & Groupe $2(30,94)$ & Groupe $1(30,11)$ & Groupe $3(35,60)$ \\
\hline $\begin{array}{l}\text { Variance expliquée }= \\
0,29\end{array}$ & Moyenne $=37,92$ & Moyenne $=38,50$ & Moyenne $=35,89$ & Moyenne $=39,48$ \\
\hline
\end{tabular}

La dimension « gouvernement d'entreprise » est traitée dans la première hypothèse, selon laquelle les entreprises relevant du capitalisme libéral de marché sont plus performantes que les autres. Nos résultats confirment cette hypothèse (tableau 3). La France et le modèle méditerranéen occupant les deux derniers rangs, cela confirme de fait l'influence des caractéristiques des systèmes économiques et sociaux dont les disparités ont été maintes fois soulignées par la littérature traitant des problématiques de gouvernement d'entreprise (Buhr et Freedman, 2001; Nobes, 1998; Roberts, 1995; Gray, 
1988). Par ailleurs, en contrôlant pour l'effet du secteur d'activité, la variance expliquée du score de gouvernement d'entreprise par le pays est très satisfaisante $\left(\mathrm{R}^{2}\right.$ ajusté $\left.=0,51\right)$. La deuxième hypothèse concerne la dimension « engagement sociétal ». Elle indique que les entreprises relevant du capitalisme libéral de marché s'impliquent davantage vis-à-vis de la société civile et sont donc plus performantes que les entreprises appartenant aux autres systèmes économiques et sociaux. En considérant le score global, les résultats permettent donc de valider cette hypothèse puisque les pays anglo-saxons se situent au premier rang du classement (tableau 4). Les résultats obtenus sont toutefois différents lorsqu'on effectue l'analyse avec le score $\mathrm{R}$ puisque les entreprises du système social-démocrate améliorent leur rang.

Tableau 5 - Résultats de l'Anova mettant en évidence l'effet du pays sur les scores du comportement sur les marchés

\begin{tabular}{|l|c|c|c|c|}
\hline \multicolumn{1}{|c|}{ Effet du pays } & Score global & Score L & Score D & Score R \\
\hline $\begin{array}{l}\text { Somme des carrés }= \\
3257,33\end{array}$ & Groupe 5 (38,31) & Groupe 5 (40,60) & Groupe 5 (27,99) & Groupe 5 (46,29) \\
\hline Degré de liberté $=4$ & Groupe 4 (35,31) & Groupe 4 (36,42) & Groupe 4 (26,46) & Groupe 4 (42,72) \\
\hline $\begin{array}{l}\text { Carrés moyens }= \\
814,33\end{array}$ & Groupe 2 (33,35) & Groupe 1 (35,74) & Groupe 2 (23,46) & Groupe 2 (41,19) \\
\hline Test en F =4,11 & Groupe 1 (31,78) & Groupe 2 (35,52) & Groupe 3 (20,19) & Groupe 3 (40,36) \\
\hline Significativité $=0,00$ & Groupe 3 (30,02) & Groupe 3 (29,19) & Groupe 1 (19,93) & Groupe 1 (39,11) \\
\hline $\begin{array}{l}\text { Variance expliquée }= \\
0,27\end{array}$ & Moyenne $=34,94$ & Moyenne = 36,68 & Moyenne = 24,96 & Moyenne = 43,03 \\
\hline
\end{tabular}

Selon la troisième hypothèse, les entreprises relevant des capitalismes socialdémocrate et méditerranéen sont supposées être plus performantes en termes de RSE sur la dimension «comportement sur les marchés» que les entreprises relevant du capitalisme libéral de marché. Cette hypothèse n'est pas vérifiée (tableau 5). Bien au contraire, les groupes 3 (capitalisme méditerranéen) et 1 (capitalisme social-démo- crate) affichent les scores globaux les plus faibles (respectivement 30,02 et 31,78). Par conséquent, les mécanismes hors marché, notamment la recherche de coopération durable entre les entreprises et leurs fournisseurs, ne semblent pas fonctionner. Toutefois, il est à noter que les entreprises relevant du modèle européen continental se situent effectivement dans une situation intermédiaire comme cela avait été postulé. 
Tableau 6 - Résultats de l'Anova mettant en évidence l'effet du pays sur les scores de gestion des ressources humaines

\begin{tabular}{|l|c|c|c|c|}
\hline \multicolumn{1}{|c|}{ Effet du pays } & Score global & Score L & Score D & Score R \\
\hline $\begin{array}{l}\text { Somme des carrés }= \\
\text { 3658,81 }\end{array}$ & Groupe 4 (40,16) & Groupe 4 (42,88) & Groupe 4 (36,55) & Groupe 4 (37,16) \\
\hline Degré de liberté $=4$ & Groupe 1 (37,33) & Groupe 1 (41,22) & Groupe 2 (35,42) & Groupe 3 (33,43) \\
\hline $\begin{array}{l}\text { Carrés moyens }= \\
914,70\end{array}$ & Groupe 2 (37,22) & Groupe 2 (40,54) & Groupe 1 (34,74) & Groupe 2 (31,38) \\
\hline Test en F =4,51 & Groupe 5 (36,21) & Groupe 5 (39,99) & Groupe 5 (33,75) & Groupe 5 (31,27) \\
\hline Significativité $=0,00$ & Groupe 3 (35,17) & Groupe 3 (36,81) & Groupe 3 (32,50) & Groupe 1 (30,81) \\
\hline $\begin{array}{l}\text { Variance expliquée }= \\
\text { 0,31 }\end{array}$ & Moyenne = 37,22 & Moyenne = 40,40 & Moyenne = 34,62 & Moyenne = 32,79 \\
\hline
\end{tabular}

Tableau 7 - Résultats de l'Anova mettant en évidence l'effet du pays sur les scores des droits de l'homme

\begin{tabular}{|l|c|c|c|c|}
\hline \multicolumn{1}{|c|}{ Effet du pays } & Score global & Score L & Score D & Score R \\
\hline $\begin{array}{l}\text { Somme des carrés }= \\
2317,62\end{array}$ & Groupe 1 (41,63) & Groupe 5 (39,26) & Groupe 1 (35,00) & Groupe 4 (52,61) \\
\hline Degré de liberté $=4$ & Groupe 5 (40,94) & Groupe 1 (37,41) & Groupe 5 (32,67) & Groupe 3 (52,38) \\
\hline $\begin{array}{l}\text { Carrés moyens }= \\
579,40\end{array}$ & Groupe 4 (39,15) & Groupe 4 (34,72) & Groupe 2 (32,32) & Groupe 1 (52,19) \\
\hline Test en F =3,15 & Groupe 2 (38,61) & Groupe 2 (32,35) & Groupe 4 (30,01) & Groupe 2 (51,10) \\
\hline Significativité $=0,01$ & Groupe 3 (34,55) & Groupe 3 (26,62) & Groupe 3 (24,52) & Groupe 5 (50,82) \\
\hline $\begin{array}{l}\text { Variance expliquée }= \\
0,32\end{array}$ & Moyenne $=39,26$ & Moyenne = 34,96 & Moyenne = 31,15 & Moyenne = 51,58 \\
\hline
\end{tabular}

Dans la quatrième hypothèse, nous indiquions que les entreprises relevant du capitalisme européen continental et du capitalisme social-démocrate sont plus performantes que les autres sur les dimensions « ressources humaines » et « droits humains ». Même si le classement des pays diffère en fonction des dimensions considérées, cette hypothèse est partiellement vérifiée: les pays des groupes 1 (modèle social-démocrate), 2 et 4 (modèle européen continental) occupent les trois premières places du classement. 
Tableau 8 - Résultats de l'Anova mettant en évidence l'effet du pays sur les scores $\mathrm{du}$ respect de l'environnement

\begin{tabular}{|l|c|c|c|c|}
\hline \multicolumn{1}{|c|}{ Effet du pays } & Score global & Score L & Score D & Score R \\
\hline $\begin{array}{l}\text { Somme des carrés }= \\
4675,52\end{array}$ & Groupe 5 (38,43) & Groupe 5 $(45,71)$ & Groupe 2 (39,30) & Groupe 1 (23,15) \\
\hline Degré de liberté $=4$ & Groupe 1 (35,56) & Groupe 1 (38,59) & Groupe 1 (37,89) & Groupe 5 (21,95) \\
\hline $\begin{array}{l}\text { Carrés moyens }= \\
1168,88\end{array}$ & Groupe 2 (34,86) & Groupe 2 (37,52) & Groupe 5 (37,66) & Groupe 2 (21,13) \\
\hline Test en F =3,36 & Groupe 3 (28,24) & Groupe 3 (35,62) & Groupe 4 (28,03) & Groupe 3 (13,79) \\
\hline Significativité $=0,01$ & Groupe 4 (26,57) & Groupe 4 (31,10) & Groupe 3 (27,55) & Groupe 4 (12,70) \\
\hline $\begin{array}{l}\text { Variance expliquée }= \\
0,26\end{array}$ & Moyenne $=33,55$ & Moyenne = 38,90 & Moyenne = 34,66 & Moyenne = 18,84 \\
\hline
\end{tabular}

Le classement obtenu pour la dimension « Environnement », développée dans la cinquième hypothèse, est quasiment identique à celui concernant le gouvernement d'entreprise. Au premier rang, se trouvent les pays du système libéral de marché; ce qui démontre que les conséquences financières des risques environnementaux ne sont pas négligeables et sont de ce fait intégrées dans l'évaluation des entreprises. La seconde place est occupée par les pays du modèle social-démocrate, témoignant ainsi de l'importance que les pays du Nord accordent à l'environnement.

\section{CONCLUSION}

L'objet de cette recherche portait sur les conséquences de la diversité des systèmes économiques et sociaux. À partir de la typologie des modèles de capitalisme développée par Amable (2005), nous avons identifié cinq groupes de pays au sein desquels nous nous sommes intéressés aux spécificités de la PSE. Les différents modèles théoriques ont montré que la PSE dépend de la formulation et de l'intégration des attentes des différentes parties prenantes de l'entreprise. Aussi, nous avons postulé que les résultats de la PSE sont en partie liés à l'appartenance de l'entreprise à un système économique et social et que ces résultats renforcent les déterminants des systèmes économiques et sociaux. Notre analyse, menée sur les six dimensions de la PSE telles que mesurées par l'agence européenne Vigeo, valide ces hypothèses pour cinq dimensions sur six. Ces résultats démontrent donc l'influence de la nature du système économique et social sur la PSE, confirmant ainsi l'importance de ce facteur pour expliquer les différences de performances et de notations sociétales. Si l'on se réfère à la typologie de la légitimité développée par Suchman (1995), qui distingue les légitimités pragmatique, morale et cognitive, le lien entre PSE et les systèmes de capitalisme repose essentiellement sur 
une légitimité morale. Lorsque la PSE intègre une dimension "gouvernement d'entreprise» et une dimension «environnement» importantes, la recherche d'une légitimité pragmatique peut apparaître. Ces résultats doivent être nuancés dans la mesure où d'une part, les spécificités de la PSE ont été présentées sur la base de la typologie proposée par Amable (2005) dont une des limites est qu'un pays donné ne relève pas systématiquement du modèle pour chaque dimension testée. D'autre part, dans cette étude, la PSE est appréhendée à partir du modèle développé par l'agence Vigeo, induisant ainsi une contingence de la mesure.

\section{Bibliographie}

Acquier A., Gond J.P., Igalens J., « Des fondements de la responsabilité sociale à la responsabilité sociale comme religion », Actes du XIVe Congrès de l'AGRH, Paris, 2005.

Agle B. R., Kelley P.C., "Ensuring validity in the measurement of corporate social performance: lessons from corporate united way and PAC campaigns", Journal of Business Ethics, vol. 31, 2001, p. 271-284.

Albert M., Capitalisme contre capitalisme, Paris, Éditions du Seuil, 1991.

Amable B., Les cinq capitalismes, Paris, Éditions du Seuil, 2005.

Amable B., Barré R., Boyer R., Les systèmes d'innovation à l'ère de la globalisation, Paris, Economica, 1997.

Aoki M., "Towards a comparative institutional analysis", Japanese Economic Review, vol. 47, n 1, 1995, p. 1-19.

Barnett M., "Stakeholder influence capacity and the variability of financial returns to corporate social responsibility", Academy of Management Review, vol. 32, n 3, 2007, p. 794816.

Buhr N., Freedman M., "Culture, institutional factors and differences in environmental disclosure between Canada and the united states", Critical Perspectives on Accounting, vol. 12, 2001, p. 293-322.

Cummings L.S., "The financial performance of ethical investment trusts: an Australian perspective", Journal of Business Ethics, vol. 25, n 1, 2000, p. 79-92.

Déjean F., Gond J. P., « Responsabilité sociétale de l'entreprise: enjeux stratégiques et méthodologies de recherche », Finance, Contrôle, Stratégie, vol. 57, n 6, 2004, p. 741-764.

Freeman R., Strategic management: a stakeholder perspective, Boston Pitma, 1984.

Giddens A., La constitution de la société, Paris, PUF, 1987.

Gond J.P., Contribution à l'étude du concept de performance sociétale de l'entreprise: fondements théoriques, construction sociale, impact financier, Thèse de doctorat en sciences de Gestion, université Toulouse 1, 2006.

Gray S. J., "Towards a theory of cultural influence on the development of accounting systems internationally", Abacus, vol. 24, n 1, 1988, p. 1-15.

Griffin J. J., Mahon J. F., "The corporate social performance and corporate financial performance debate", Business and Society, vol. 36, n 1, 1997, p. 5-31. 
Hall P., Soskice D. (dir.), Varieties of capitalism: the institutional foundations of comparative advantage, Oxford, Oxford University Press, 2001.

Igalens J., Gond J.P., "Measuring Corporate Social Performance in France: a critical and empirical analysis of ARESE data", Journal of Business Ethics, vol. 56, 2005, p. 131-148.

Johnson R. A., Greening D. W., "The effects of corporate governance and institutional ownership types on corporate social performance", Academy of Management Journal, vol. 42, $\mathrm{n}^{\circ} 5,1999$, p. 564-576.

Jones M. T., "Missing the forest for the trees. A critique for the social responsibility concept and discourse", Business and Society, vol. 35, n 1, 1996, p. 7-41.

Long B. S., Driscoll C., "Codes of ethics and the pursuit of organizational legitimacy: Theoretical and empirical contributions", Journal of Business Ethics, vol. 77, 2008, p. 173-189.

Maati J., Le gouvernement d'entreprise, Paris - Bruxelles, De Boeck, 1999.

Margolis J. D., Walsh J. P., "Misery loves companies: wither social initiatives by business?", Administrative Science Quarterly, vol. 48, 2003, p. 268-305.

Nobes C., "Towards a general model of the reasons for international differences in financial reporting", Abacus, vol. 34, n 2, 1998, p. 162-187.

North D., Institutions, institutional change and economic performance, Cambridge, Cambridge University Press 1990.

Pasquero J., « La responsabilité sociale de l'entreprise comme objet des sciences de gestion: le concept et sa portée », Responsabilité sociale et environnementale de l'entreprise, M.F. Turcotte et A. Salmon (eds.), Presses de l'Université du Québec, 2005, p. 112-143.

Pasquero J., « Fusions et acquisitions: principes d'analyse éthique », Revue française de Gestion, ${ }^{\circ} 74,1989$, p. 97-108.

Roberts A., "The very idea of classification in international accounting", Accounting Organizations and Society, vol. 20, $\mathrm{n}^{\circ} 7-8,1995, \mathrm{p} .639-664$.

Rowley T., Berman S., "A brand new brand of corporate social performance", Business and Society, vol. 39, n 4, 2000, p. 397-419.

Ruf B. M., Muralidhar K., Paul K., "The development of a systematic, aggregate measure of corporate social performance", Journal of Management, vol. 24, n 1, 1998, p. 119-133.

Sharfman D., "The construct validity of the KLD social performance data ratings", Journal of Business Ethics, vol.15, n 3, 1996, p. 287-297.

Teoh S.H., Welch I., Wazzan C.P., "The effect of socially activist investment policies on the financial markets: evidence from the South African boycott", Journal of Business, vol. 72, $\mathrm{n}^{\circ} 1,1999$, p. 35-89.

Wood D. J., "Corporate social performance revisited", Academy of Management Review, vol. $16, \mathrm{n}^{\circ} 4,1991$, p. 691-718.

Wood D.J., Jones R.E., "Stakeholder mismatching: a theoretical problem in empirical research in corporate social performance", International Journal of Organizational Analysis, vol. 3, 1995, p. 229-267. 
